\title{
Redes sociales e innovación en el sector de la cultura
}

\author{
Social Networks and \\ Innovation in Culture
}

\section{María Isabel Camio (Argentina)}

Doctora en Administración

Centro de Estudios en Administración

camio@econ.unicen.edu.ar

\author{
Constanza María Díaz Bilotto (Argentina) \\ Centro de Estudios en Administración \\ constanza.diazbilotto@gmail.com
}

\section{Resumen}

Este artículo de revisión explora diferentes abordajes teóricos acerca de la industria creativa y cultural y señala el papel de los agrupamientos productivos en la creación y el fortalecimiento de organizaciones innovadoras. Se expone la metodología de análisis de redes aplicada al mapeo de las relaciones de los actores clave del Polo de Industria

\section{Jesús Estefanía Rivas Urrutibehety \\ (Argentina) \\ Centro de Estudios en Administración estefaniarivasu@gmail.com}

Para Citar este artículo / to Cite this article Camio, M. I.; Díaz Bilotto, C. M. y Rivas Urrutibehety, J. E. (2014). Redes sociales e innovación en el sector de la cultura, Poliantea, 10(18), pp. 271-302. 
Cultural y Arte Creativo de la Universidad Nacional del Centro de la Provincia de Buenos Aires (Argentina). A partir del análisis, se identifica la necesidad de generar lazos transversales y alianzas institucionales para el crecimiento y desarrollo del sector y sus actividades. Se identificó la existencia de capacidades para la generación y difusión de la innovación, lo cual se relaciona con una etapa avanzada dentro del desarrollo embrionario del referido clúster.

Palabras clave: redes, innovación, industria cultural, arte creativo.
[Cultural Industry and Creative Art Pole] of the Universidad Nacional del Centro de la Provincia de Buenos Aires (Argentina) [National University of the Center of Buenos Aires Province]. From this analysis, we were able to identify the need to generate alliances among institutions to enable the growth and development of the sector and its activities. We also recognized the existence of capacities to generate and promote innovation, which relates to an advanced stage of the development of this cluster.

Keywords: networks, innovation, cultural industry, creative art. 


\section{Redes sociales e innovación en el sector de la cultura}

\section{Social Networks and Innovation in Culture}

\section{María Isabel Camio (Argentina) Doctora en Administración Centro de Estudios en Administración camio@econ.unicen.edu.ar}

\author{
Constanza María Díaz Bilotto (Argentina) \\ Centro de Estudios en Administración \\ constanza.diazbilotto@gmail.com
}

\author{
Jesús Estefanía Rivas Urrutibehety \\ (Argentina) \\ Centro de Estudios en Administración \\ estefaniarivasu@gmail.com
}

\section{Introducción}

En el presente artículo se exponen diferentes abordajes teóricos acerca de la industria creativa en general y cultural en particular.

En el estudio del fenómeno de la innovación se hace hincapié en no solo el análisis en la empresa, sino también en el abordaje del conjunto productivo del sector o de fragmentos de ese conjunto. Las particularidades de la industria cultural requieren identificar elementos distintivos en el fenómeno de la innovación organizacional.

La importancia de los clústeres regionales resulta destacada recpecto de su capacidad para favorecer sinergias e intercambios productivos entre empresas e instituciones para el desarrollo de innovaciones sostenidas.

En términos conceptuales, se exponen los aspectos de la metodología de análisis de redes relevantes para el presente trabajo.

En el desarrollo del artículo se exponen los aspectos específicos que contextualizan el sector en estudio en su territorio, se identifican las instituciones 
intervinientes y sus niveles y se expone la red que incluye ambas relaciones en análisis.

En términos metodológicos, se transitan dos instancias en el proceso de recolección de datos. En una primera etapa, las entrevistas en profundidad de los actores clave, y en una segunda fase las particularidades de la dimensiones de análisis estructural y relacional del estudio de redes. Se particulariza el estudio de las relaciones respecto de la información circulante y existencia o potencial para la generación de alianzas, a partir de su contribución al desarrollo de la innovación organizacional.

Se focaliza el estudio de las relaciones respecto de los indicadores de centralidad de grado y de centralidad por intermediación. Se identifican las fortalezas de los lazos y la identificación de agujeros estructurales para la generación de innovación.

\section{Marco teórico}

\section{Industrias creativas y culturales}

La definición de industria cultural no es única, pero en general se puede decir que hace referencia a la industrialización de la cultura originada desde comienzos del siglo pasado. Introducirse en la temática obliga a la conceptualización misma de la "cultura", así como de "productos culturales", "productos culturales masivos", "economía cultural", "industrias creativas" e "industrias del ocio", entre otros, y su relación con la economía agregada de una región, país o en el ámbito internacional.

Power (2011) define las industrias culturales y creativas como aquellas ocupadas en la creación y provisión de productos de mercado (bienes, servicios, actividades), los cuales dependen de insumos creativos y culturales para tener valor.

Las industrias culturales y creativas comprenden, por tanto, aquellas empresas principalmente con fines de lucro que se ocupan de la creación, producción o distribución de bienes y servicios culturales-creativos. Otro núcleo común de las actividades económicas de las industrias culturales y creativas es un proceso creativo que incluye cualquier acto artístico, literario, cultural o arquitectónico para el desarrollo de productos o servicios (Kind y Meier zu Köcker, 2013).

La industria creativa puede ser definida como los ciclos de creación, producción y distribución de bienes y servicios que usan la creatividad y el capital intelectual como insumos primarios (Unctad [United Nations Conference on Trade and 
Development] 'Conferencia de las Naciones Unidas sobre Comercio y Desarrollo', 2008), o bien, como dice el Department for Culture, Media \& Sport (DCMS 'Departamento de Cultura, Medios y Deporte del Reino Unido'), puede ser entendida como aquellas industrias con el potencial de crear ganancias y puestos de trabajo mediante el desarrollo de la propiedad intelectual.

La idea de industrias creativas enfatiza el papel central de la creatividad (proceso mental y social de descubrir nuevas ideas, conceptos o asociaciones) en cómo las firmas y los trabajadores proponen comercializar sus productos. Estos tipos de productos ofrecidos por las industrias creativas pueden ser valorados tanto como tales o como servicios basados en el conocimiento (Power, 2011).

Las industrias creativas contienen a las denominadas industrias culturales, caracterizadas por la economía de la cultura, y a otras industrias cuyo componente principal sea la creatividad (Rodríguez, 2009, citado por García Domínguez, 2010).

La Unctad (2008) hace una distinción entre "actividades corriente arriba" (actividades culturales tradicionales, como las artes escénicas o visuales) y "actividades corriente abajo" (más cercanas al mercado, como la publicidad, o las actividades relacionadas con los medios). Desde esta perspectiva, las industrias culturales son un subsegmento de las industrias creativas.

Para Stoneman (2010), el sector cultural se divide en tres subsectores: las artes (galerías, arquitectura, diseño y artesanías), las industrias culturales (productos audiovisuales y libros, prensa, artes escénicas y artes visuales) y los deportes y el turismo.

Para la Unctad (2008), la economía cultural es la aplicación del análisis económico a las artes creativas, escénicas e industrias culturales, ya sean actividades públicas, ya sean privadas.

Getino (2004) introduce en su estudio de la industria cultural el concepto de economía cultural al reconocer que la cultura es también economía cuando genera y representa inversiones, producción, ventas, balanza comercial, gasto público y gasto privado, empleo y pagos por derechos de autor, entre otras variables de análisis.

La dimensión económica de la cultura salta a la vista cada vez más a través de estudios e investigaciones realizados por organismos intergubernamentales o por expertos 
de distintas procedencias. Pero no se trata de reducir el estudio de la economía de la cultura encarando solo su incidencia en el crecimiento económico y el empleo. Lo es también, y fundamentalmente, para los procesos de integración nacional y regional, además de lo que puede significar para la identidad y el autorreconocimiento de los individuos y las sociedades (Getino, 2004).

Para Del Corral (2005), las industrias culturales aportan un valor añadido a los contenidos, al tiempo que construyen y difunden valores culturales de interés individual y colectivo. Las industrias culturales tienen también una imperiosa necesidad de innovación constante y una estructura económica particular; el núcleo ineludible de su negocio consiste en transformar contenidos culturales - valores simbólicos-en valor económico.

Al mismo tiempo, en la "sociedad o economía del conocimiento", el conocimiento adquiere una importancia determinante como factor clave para el desarrollo productivo y la integración socioeconómica.

\section{Innovación en industrias}

\section{culfurales}

Hoy en día, la innovación es considerada una capacidad dinámica. Este concepto hace referencia a "la capacidad de la organización de crear, extender o modificar su base de recursos intencionalmente" (Helfat etal., 2007, p. 4) por la adición de nuevo conocimiento en los nuevos productos, servicios, procesos, tecnologías o métodos de gestión. Siendo necesarias en ambientes de negocios de alta tasa de cambio, abiertos a la competencia global, las capacidades dinámicas son la base para las ventajas competitivas y la creación de valor (Teece, 2007).

Existe consenso respecto de la creciente importancia de la innovación como proceso que facilita la competitividad empresarial y que contribuye al desarrollo económico de las sociedades (Lundvall y Maskell, 2000; Yoguel y Boscherini, 1996).

Según Stoneman (2010), en las industrias culturales tienen lugar innovaciones tecnológicas del tipo tradicional y funcional, pero también aparece el tipo de innovación al cual el autor denomina soft innovation. Esta está concentrada en cambios de productos (o procesos) de naturaleza estética o intelectual. Asimismo, 
si bien este tipo de innovación es natural en el sector cultural, ya que sus productos son de naturaleza estética, puede ser detectado en otras industrias en las cuales las características funcionales de sus productos o servicios son afirmadas a través de aspectos estéticos.

Borello y González (2013) proponen en su estudio sobre la innovación y las formas de organización de la industria audiovisual de la Argentina un abordaje de la innovación desde dos instancias: el conjunto productivo o fragmentos de ese conjunto y las empresas individuales. En el plano del conjunto productivo, examinan los procesos de innovación distribuida que acompañaron la creación de un nuevo movimiento estético en el cine argentino (nuevo cine argentino), y en las empresas estudian tres planos de los procesos de innovación: la introducción de nuevos productos o servicios y el uso de nuevas tecnologías, los productos y otros indicadores de apropiabilidad de las actividades de innovación, las fuentes de información y la percepción de los empresarios respecto de si sus productos tienen una estética que los distinga de los otros.

Para Borello y González (2013), en la producción audiovisual, y presumiblemente en otras industrias culturales, hay procesos distribuidos y colectivos de creación y de desarrollo de innovaciones que se asocian a la generación de nuevos géneros y de revoluciones estéticas.

Esta observación guarda relación con el modelo de innovación denominado innovación abierta, que nace del reconocimiento de la importancia de las redes mediante las cuales las organizaciones se relacionan con su entorno, y es el tipo de innovación que permite que la propiedad intelectual, las ideas y la gente fluyan libremente dentro y fuera de la organización. Hoy en día, el flujo hacia organizaciones de contribuciones externas recibe mayor atención (outside-in open innovation). Sin embargo, el proceso de invertir en proyectos generadores de innovación fuera de la organización (inside-out open innovation) tiene sus beneficios al ahorrar costos de $\mathrm{I}+\mathrm{D}$, potenciar relaciones con proveedores y relaciones asociativas y promover ecosistemas innovadores (Chesbrough y Garman, 2009).

Miles y Green (2008) han realizado una compilación de estudios referidos a la innovación en industrias creativas, concluyendo que han sido pocos los intentos de explorar la innovación estética y de contenidos. 
Handke (2004), citado por Miles y Green (2008), ve las industrias culturales como caracterizadas por su "contenido creativo", concepto cercano al soft innovation de Stoneman (2010). En su estudio, contrasta este tipo de innovación con la "innovación monótona-tradicional-tecnológica”.

Tanto Stoneman (2010) como Handke (2004), citado por Miles y Green (2008), apuntan hacia innovaciones que involucran contenido, estéticas o experiencias. Mientras que Stoneman (2010) nota que las definiciones estándar del Manual de Oslo, principalmente orientadas a innovaciones tecnológicas, dependen de la funcionalidad como una medida de identificación de significatividad de la innovación, él y Handke proponen que el impacto en el mercado puede ser una métrica útil y fácilmente asequible para medir la significatividad de las innovaciones estéticas, sin olvidar la diferencia entre el valor económico y cultural de las producciones culturales y creativas.

Bakhshi y Throsby (2009) plantean el objetivo de examinar el concepto de innovación y su relación con las instituciones culturales. Estos autores identifican cuatro aspectos de la innovación que pueden ser llevadas a cabo por las instituciones culturales a través de las artes creativas: innovación en extender el alcance de audiencia, innovación en el desarrollo de la forma del arte, innovación en la creación de valor e innovación en la gestión empresarial.

\section{Clústeres}

A partir de incluir en la dimensión económica el análisis de los procesos de integración regional y a la luz de modelos de innovación abierta, resulta necesario estudiar las redes entre empresas-instituciones del sector cultural.

Los clústeres se definen como concentraciones geográficas de compañías interconectadas, proveedores especializados, abastecedores de servicios, firmas en industrias relacionadas e instituciones asociadas en campos particulares que compiten pero también cooperan (Porter, 1998).

En la literatura internacional, se reconoce que la creatividad frecuentemente se caracterizada por la aglomeración de firmas, de modo que las industrias creativas no se distribuyen homogéneamente en un territorio, sino que se concentran en un espacio (Cooke y Lazzeretti, 2008; Scott, 2005). De hecho, las industrias creativas tienden a formar clústeres en ciudades y dirigirse a gran variedad 
de sectores económicos (Maskell y Lorenzen, 2004, citados por Lazzeretti, Boix y Capone, 2009).

Camagni, Maillat y Matteaccioli (2004), Hoover y Vernon (1959), Nooteboom (2000), Glaeser (2000), Florida (2002), citados por Lazzaretti, Boix y Capone (2009), sugieren cinco hipótesis como determinantes de la formación de clústeres en las industrias creativas:

- por la presencia de herencia histórica y cultural;

- el efecto de las economías de escala y la organización industrial;

- el concepto de variedad relacionada y su contribución a las actividades creativas;

- el papel del capital humano;

- la teoría de Florida (2002) acerca de las clases creativas.

La administración de clústeres puede servir como una estructura organizacional de soporte a la cooperación y comunicación entre sectores de las industrias culturales, a través de sectores, y también con sectores fuera de estas industrias. Esto se debe a la heterogeneidad de sectores y a su naturaleza transversal. Esta cuestión se condice con la idea de que la cooperación entre sectores y los proyectos transdisciplinarios promueven potenciales sinergias e innovaciones (Kind y Meier zu Köcker, 2013).

La importancia de los clústeres regionales radica en su capacidad para favorecer sinergias e intercambios productivos entre las empresas e instituciones, además, suelen impactar positivamente respecto de la creación de empresas innovadoras, de vinculación con centros especializados de investigación y desarrollo y de revitalización de otros sectores de actividad existentes en una determinada región. La interacción social y la cooperación empresarial no solo facilitan el acceso al conocimiento codificado o explícito existente en un determinado sector de actividad o cadena de valor, sino que también $y$, fundamentalmente, ayudan a desarrollar vínculos estrechos o capital social (Nahapiet y Ghoshal, 1998).

El Tratado de Lisboa postula la relación entre innovación regional y la presencia de clústeres de industrias creativas y culturales. Dado que las industrias culturales y creativas operan en mercados de rápido desarrollo y orientados a las modas, la innovación continua y creatividad son núcleos en la ventaja competitiva. Los clústeres regionales deben innovar para sobrevivir o crecer (Power, 2011). 
En el presente artículo, se plantea la necesidad de ampliar nuestra perspectiva acerca de los tipos de intermediación que incluyen y requieren los procesos de innovación, como la construcción de redes, el sostenimiento del aprendizaje social y la gestión de la dinámica de poder y los conflictos (Leeuwis y Aarts, 2011). En este sentido, la literatura sobre políticas públicas y administración ha prestado especial atención a la conformación y desarrollo de clústeres o conglomerados de empresas basadas en el conocimiento como herramienta fundamental para el desarrollo local y regional (Florida, 2002; Koepp, 2002; Porter, 1998; Saxenian, 1994).

Como en muchos sectores industriales, empresas y trabajo son distribuidos desigualmente, y esto indica la existencia de sistemas de innovación regionales e industriales y clústeres apuntalados por condiciones regionales favorables. El empleo y la competitividad en las industrias creativas y culturales no están directamente relacionados con el tamaño del mercado laboral o la población, y pueden no ser consideradas como un subproducto de la habitación humana. Las actividades creativas y culturales son industrias impulsadas por el conocimiento, atraídas por mercados especializados y clústeres. Las escalas y los alcances de las organizaciones y proyectos de las industrias culturales son soportados por clústeres y grandes mercados de trabajo (Power, 2011).

\section{Conceptos de redes}

Una red social es un conjunto de actores (o puntos o nodos o agentes) entre los que pueden existir relaciones (o vínculos) (Hanneman, 2001).

El análisis de redes sociales ha experimentado en los últimos años una creciente popularidad en el mundo de las ciencias sociales como alternativa ( $y$ en otros casos como complemento) al análisis de tipo individualista-automista. Frente al estudio tradicional centrado en la consideración de atributos individuales y en la construcción de categorías basadas en estos atributos, el análisis de redes sociales aboga por tomar las relaciones entre actores como el "material" sobre el cual se construye y se organiza el comportamiento social de los actores (Verd Pericás y Martí Olivé, 1999).

El estudio de redes sociales es una herramienta para el estudio de las conexiones existentes. En la práctica, los tipos de redes en los cuales los teóricos de redes tienden a enfocarse pueden ser categorizados en dos grupos básicos: estados y eventos. 
Los estados tienen continuidad en el tiempo. Esto no significa que son permanentes, sino que tienen una persistencia "abierta". Los lazos de estado pueden ser dimensionalizados respecto de fuerza, intensidad y duración. En contraste, un lazo del tipo de "evento" tiene una naturaleza discreta y transitoria, y puede ser contada por periodos. Este tipo de lazos es el que los investigadores tienen en mente cuando definen redes como un "patrón recurrente de lazos" (Dubini y Aldrich, 1991 y Ebers, 1997, citados por Borgatti y Halguin, 2011).

La unidad de análisis en la aproximación de las redes sociales no es el individuo, sino el conjunto formado por los individuos y los lazos entre ellos (Lozares, 1996). Como indica Hanneman (2001), rara vez los análisis de redes sociales diseñan muestras. Muy a menudo, identifican una población y dirigen un censo (por ejemplo incluyen todos los elementos de la población como unidades de observación). Si un actor llega a ser seleccionado, han de incluirse todos los actores con los cuales este tiene (o puede tener) relaciones. Como resultado, los enfoques de redes intentan estudiar poblaciones totales a través del censo más que a través de la muestra.

\section{Dimensiones de redes}

sociales: dimensión

estructural y relacional

El análisis de la dimensión estructural, cuando se considera la configuración de la red, se efectúa mediante la determinación de los patrones de enlaces entre sus miembros (Mayoral et al., 2012).

Burt (1980), citado por Verd Pericás y Martí Olivé (1999), menciona esta misma dimensión como el enfoque posicional, donde el actor es uno más en un sistema interconectado de actores, de modo que deben considerarse todas las relaciones en que está implicado. Es posible realizar una evaluación básica de la estructura de una red social mediante el análisis del tamaño y la densidad y de esta red y la centralidad de sus actores (Mayoral et al., 2012).

El tamaño de una red es un factor crítico para cualquier estructura de relaciones sociales, ya que los actores disponen de recursos y capacidades limitadas para construir y mantener lazos.

La medida de densidad de una red muestra hasta qué punto el entramado alcanza su potencial máximo o situación de saturación, considerando la relación que existe entre cantidad delazos presentes frente a la cantidad de lazos posibles (Díez, 2008). 
El grado de centralidad es el número de actores a los cuales un actor está directamente unido. El grado de centralidad se divide en grado de entrada (es la suma de las relaciones referidas hacia un actor por otros) y de salida (es la suma de las relaciones que los actores dicen tener con el resto), dependiendo de la dirección del flujo (Velázquez y Aguilar, 2005).

Por su parte, la dimensión relacional focaliza el papel de los lazos directos entre los actores en relación con los contenidos transaccionados en las interacciones y su diversidad. Entre los contenidos transaccionados en esta dimensión están la amistad, el intercambio de información, el respeto, la confianza, las normas, las sanciones, o el consejo y la identificación (Nahapiet y Ghoshal, 1998). La idea central de la visión relacional consiste en que el análisis no se construye a través de categorías sociales o atributos, sino a través de lazos o vínculos entre actores, incluso, no estando directamente relacionados y unidos (Wellman, 1983 y Granovetter, 1973, citados por Lozares, 1996).

En este sentido, la importancia que un actor tiene en una red puede deberse a su capacidad para controlar la comunicación entre las diversas organizaciones. Por esto, uno de los indicadores relevantes en esta dimensión es la centralidad por intermediación. Esta medida define el poder que tiene cierto nodo dentro de una red para conectar o ser "puente" entre un grupo de nodos y, en consecuencia, se identifican como nodos "bisagra" en el manejo de los recursos que puedan fluir de la red (Gaete y Vásquez, 2008), también llamado grado de cercanía por Velázquez y Aguilar (2005).

\section{Fortaleza de lazos y agujeros estructurales}

Granovetter (1973) define que la fuerza de un lazo es proporcional al tiempo compartido, la intensidad emocional del vínculo, el grado de confianza y la reciprocidad que lo caracteriza. De esta manera, establece que existen lazos fuertes y débiles dentro de las redes sociales. Uzzi (1997) retoma estos conceptos para clasificar los lazos que existen entre las organizaciones. Los lazos débiles son particularmente importantes en procesos de difusión (de ideas, información, innovaciones, etc.). Los lazos fuertes, por su parte, son más importantes en procesos de generación de innovación (Uzzi y Dunlap, 2005).

Granovetter (1973) plantea dos premisas: la primera establece que, cuanto más fuerte sea el lazo entre 
dos personas, más probable es que sus mundos sociales se superpongan, que sus lazos tengan las mismas terceras partes. La segunda premisa de Granovetter es que los lazos "puente" son una fuente potencial de ideas novedosas. Poniendo las dos premisas juntas (Borgatti y Halguin, 2011), Granovetter razona que los lazos fuertes son poco probables que se conviertan en fuentes de información novedosa. Los lazos débiles, los que tienen más posibilidades de ser puentes, son fuentes potenciales de información novedosa.

Según Ahuja (2000), citado por Gaete y Vásquez (2008, p. 33), un "agujero estructural", o "hueco estructural", hace referencia a características estructurales de las redes, específicamente cuando dentro de una red existen diversas zonas densas unidas unas a otras por escasos lazos o por algunos nodos. Estos actores alcanzan una posición central entre los actores de la red, especialmente por las ventajas de acceso a la información y conocimientos que puedan circular por la red, y sobre todo de estos núcleos densos de nodos. Respecto de las redes de conocimiento, estas posiciones se correlacionan con actividades innovadoras y competitivas; sin embargo, estos actores contarán con menor capital social integrador que aquellos que operan en las zonas más densas.

Burt (2000), citado por Ying (2010), definió los agujeros estructurales como oportunidades para obtener nuevas ventajas, acceder a información no redundante y difundir conocimiento.

\section{Materiales y métodos \\ Objetivos}

Surge como objetivo del presente artículo explorar las capacidades existentes para la generación de innovación sostenida en las organizaciones del Polo de Industria Cultural y Artes Creativas de la Universidad Nacional del Centro de la Provincia de Buenos Aires (Unicen). Se exponen los siguientes objetivos particulares:

- Explorar diferentes abordajes teóricos acerca de la industria cultural y creativa.

- Definir polos-clústeres o agrupamientos del sector e identificar sus tipologías.

- Construir un mapa de relaciones de los actores clave del Polo de Industria Cultural y Artes Creativas (organizaciones del sector de la industria cultural y artes creativas, la universidad, 
organismos públicos y privados que apoyen el sector).

- Identificar actores clave ${ }^{1}$ en el momento de impulsar iniciativas y generar vínculos para procesos de innovación sostenidos.

\section{Fuentes de recolección de datos}

En cuanto a la recolección de datos, se llevó adelante a lo largo de dos etapas. Con el objetivo de tener una primera aproximación al sector, se realizaron seis entrevistas en profundidad, no estructuradas, a 13 representantes de instituciones públicas y privadas del sector.

En función de la información recolectada durante la primera etapa, se llevaron adelante entrevistas individuales estructuradas, a través de un cuestionario, a los actores considerados focales (por su experiencia, influencia o dimensión institucional) del sector (que no necesariamente coinciden con los actores entrevistados durante la primera etapa del relevamiento).

Adicionalmente, se realizó la exploración de información secundaria (artículos periodísticos y páginas web

En este trabajo se definen los actores clave por ser los nodos respecto de los índices analizados. Estos son los de más altas centralidad de grado y de intermediación. institucionales), fundamental para completar la contextualización del sector.

En esta segunda etapa se seleccionó el método de recolección de información relacional denominado método de bola de nieve, mediante el cual se comienza focalizando un conjunto de actores, y a cada uno se les pregunta por algunos de sus lazos con otros, quienes son identificados como individuos por entrevistar en una etapa posterior.

Los primeros nodos fueron considerados la "ola cero", a los cuales se les hizo una serie de preguntas sobre sus lazos de información y generación de alianzas. De esta manera, cada actor de la ola cero debió mencionar actores (sin límites máximos ni mínimos) con los que está relacionado y conectado en su red. Los individuos que surgen de estas entrevistas pasarán a ser la segunda ola de la red (ola uno), a la cual se limita esta investigación por limitación de recursos (tiempo).

Durante la entrevista, se les pidió en primera medida que indicaran si pertenecen a una organización-empresa o, por el contrario, si tenían emprendimientos individuales. En caso de pertenecer a una organizaciónempresa, se les pidió que hicieran 
referencia a cuál, para poder así diferenciar aquellas organizaciones con presupuesto propio de aquellas que dependen de otra organización u organismo para su subsistencia. Para las organizaciones dependientes de otro ente de mayor tamaño o responsabilidad, se pidió que indicaran su nombre.

Con el cuestionario se buscó alcanzar a todos los actores considerados focales de la red a través de un censo y conocer el vínculo que los relaciona dentro de ella. Para esto, previamente se plantean dos posibles vínculos o variables de relación por analizar:

- Red de información: se refiere a aquellos individuos que le proveen distintos tipos de información a los considerados nodos focales de la red. Puede ser información sobre cómo promocionar los espectáculos, obras o eventos culturales; sobre subsidios, financiamientos o programas destinados para este sector, y también acerca de propuestas artísticas de interés para el encuestado, o bien sobre recursos técnicos para llevar adelante producciones artísticas.

- Red de alianzas: aquella red constituida por personas con las que los nodos de la ola cero llevan o llevarían adelante un emprendimiento cultural.

Para el análisis de los datos relacionales y la influencia de los tipos de vínculos en la innovación abierta del sector, se decidió unificar estas dos redes en una red resultante de "información y alianzas". Esta red representa todas las relaciones que parten de los integrantes de la ola cero con respecto a la información y colaboración.

Un aspecto por destacar de la red resultante es que permite identificar vínculos de mayor fortaleza, los cuales provienen de la mención de una misma persona en las dos redes.

\section{Métodos implementados}

Variadas investigaciones desde el abordaje de la economía (Lazzeretti, Boix y Capone, 2009; Power, 2011; Unctad, 2008) han estudiado a las industrias culturales y la economía cultural, dando lugar a reportes que presentan información en la región o el país.

En este artículo se acoge una perspectiva amplia de la economía cultural (Getino, 2004), vinculando enfoques de la sociología del trabajo y de las organizaciones y en la búsqueda 
de elementos que contribuyan al desarrollo de un contexto interorganizacional (redes-clústeres) para el fortalecimiento de iniciativas innovadoras que deben desarrollarse por emprendedores-empresas-organizaciones del sector de arte creativo en el territorio en estudio.

Se analizaron tanto la dimensión estructural como la relacional dentro de la red del sector. Con la primera se buscó conocer la proximidad que existe entre cada uno de los nodos para identificar cuán accesible es un determinado actor para los demás actores de la red, así como también la saturación entre los lazos, teniendo en cuenta la cantidad de lazos presentes, frente a los lazos posibles. Con la dimensión relacional se buscó focalizar cuál era el papel de los actores en la red con respecto a los demás nodos a través de las distintas variables antes mencionadas (información y alianzas).

En la dimensión estructural, se incluye el análisis de densidad y el de centralidad. La medida de densidad de una red muestra hasta qué punto el entramado alcanza su potencial máximo o situación de saturación considerando la relación que existe entre cantidad de lazos presentes frente a la cantidad de lazos posibles (Díez, 2008).
Se analiza el grado de centralidad (número de actores a los cuales un actor está directamente unido), específicamente en grado de entrada (Velázquez y Aguilar, 2005). Se estudia la medida de centralidad por intermediación (Brandes, 2001), la que define el poder que tiene cierto nodo dentro de una red para conectar o ser "puente" entre pares de nodos y, en consecuencia, se identifican como nodos "bisagra" en el manejo de los recursos que puedan fluir por la red (Gaete y Vásquez, 2008), también llamado grado de cercanía por Velázquez y Aguilar (2005).

Los nombres de los entrevistados se codificaron como E\#, mientras que las personas mencionadas por estos (ola uno) se codificaron como $\mathrm{n} \# \#$. Cabe recordar que la red que se expone en el apartado "Descripción de las redes en análisis" resulta de la combinación de todas las menciones de los individuos de la ola cero, sin distinción acerca de si el lazo corresponde a una relación de información o de colaboración. Los únicos lazos que se diferencian de los demás (con grosores diferentes) son los que unen a los entrevistados con las personas con quienes dicen compartir tanto información como colaboración. 
En la tabla 1 se exponen las ex- número máximo de enlaces, densipresiones matemáticas utilizadas para dad, centralidad de grado, centralicalcular los principales parámetros de dad de grado de entrada, centralidad las redes sociales: tamaño de la red, de intermediación.

Tabla 1. Expresiones matemáticas utilizadas para calcular los principales parámetros de las redes sociales

Tamaño de la red

Donde $g g$ es la cantidad de nodos del grafo

\section{$g$}

Número máximo de enlaces que puede
tener un grafo de tamaño $g g$

Densidad

Donde $L L$ son la cantidad de lazos

$$
\Delta=\frac{L}{g(g-1) / 2}=\frac{2 L}{g(g-1)}
$$

presentes

Centralidad de grado

Donde $d\left(v_{\mathrm{i}}\right) d\left(v_{\mathrm{i}}\right)$ es el grado de $v_{\mathrm{i}} v_{\mathrm{i}}$

$$
C_{D}\left(v_{i}\right)=d\left(v_{i}\right)=x_{i}+=\sum_{j} x_{i j}=\sum_{j} x_{j i}
$$

Centralidad de grado de entrada

$$
C_{D}^{i n}\left(v_{i}\right)=d^{i n}\left(v_{i}\right)=x_{i}+=\sum_{j} x_{j i}
$$

Centralidad de intermediación

Donde $g_{j k} g_{j k}$ es la cantidad de caminos

conectando $v_{j} v_{j} y v_{k} v_{k}, y$

$g_{j k}\left(v_{\mathrm{i}}\right) g_{j k}\left(v_{\mathrm{i}}\right)$ es la cantidad de caminos

$$
\sum_{j\langle k} \frac{g_{j k}\left(v_{i}\right)}{g_{j k}}
$$

que conectan $v_{j} v_{j} y v_{k} v_{k}$ en los cuales

participa $v_{\mathrm{i}} v_{\mathrm{i}}$ 


\section{Resultados}

\section{Contextualización específica}

Como antecedente por destacar, el 10 de diciembre de 2010 se crea como iniciativa de la Unicen el Polo de Industria Cultural y Artes Creativas. $\mathrm{Su}$ objetivo es consolidar la industria, apoyar y asesorar a los emprendedores del sector, crear sinergias y fomentar la salida laboral de los estudiantes de las carreras de Teatro y Realización Integral de Artes Audiovisuales de la Facultad de Arte, y Comunicación Social de la Facultad de Ciencias Sociales. Las áreas por desarrollar y apoyar incluyen animación, diseño, pre y posproducción, música y guiones, entre otros.

Se suma a la generación de recursos humanos calificados egresados de la Unicen que ella es cabecera del Polo de la Provincia de Buenos Aires ${ }^{2}$ para la generación de contenidos para la televisión digital (Polo Audiovisual Tecnológico). La Unicen está a cargo

2 El Programa Polo Audiovisual Tecnológico está en funcionamiento desde el 29 de junio de 201 1, al firmarse el Convenio de Cooperación y Asistencia Técnica entre el Ministerio de Planificación Federal, Inversión Pública y el Consejo Interuniversitario Nacional. A partir de la división del país en nueve regiones (polos), se constituye un sistema federal en red donde las universidades nacionales "nuclean" a los actores del sector audiovisual televisivo de cada comunidad para conformar nodos. de la administración y concentración de los contenidos generados por cada nodo emplazado en la región, los que no solo estarán integrados por entidades académicas, sino también por cooperativas y organizaciones sociales afines al sector audiovisual, pymes audiovisuales, productores independientes, televisoras comunitarias y organismos públicos locales.

Asimismo, se reconocen otras instituciones, ya sean públicas, ya sean privadas, que si bien no forman parte de la Unicen, influyen en el desarrollo del sector cultural, por lo que también serán tenidas en cuenta en el presente artículo.

En la figura 1 y en la tabla 2 se presenta la información sobre las instituciones a las que pertenecen cada uno de los sujetos de la ola cero, así como el papel o cargo desempeñado. Como se puede ver, este conjunto de personas entrevistadas pertenecen a dos tipos de instituciones: públicas y privadas, las cuales, a su vez, se agrupan en otra serie de instituciones. 


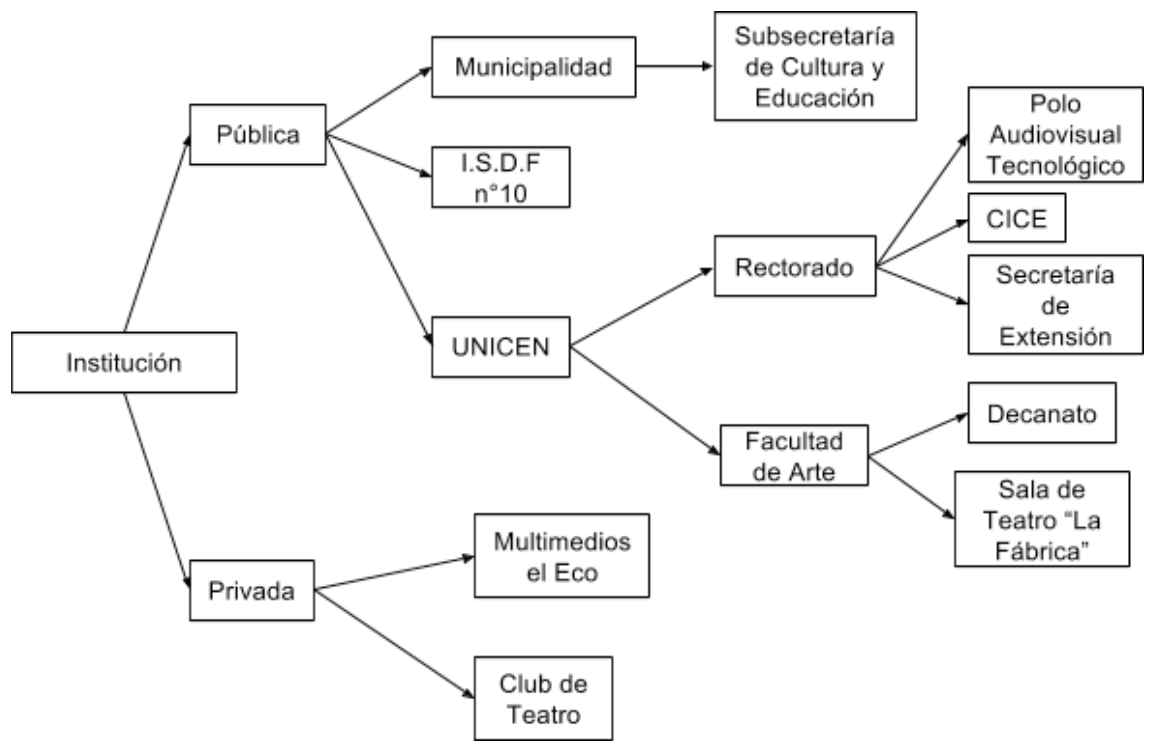

Figura 1. Ola cero: instituciones incluidas.

Tabla 2. Papel institucional de los encuestados en la ola cero

\begin{tabular}{|c|c|c|c|}
\hline $\begin{array}{c}\text { Código de } \\
\text { entrevistado }\end{array}$ & $\begin{array}{c}\text { Institución a la que } \\
\text { pertenece }\end{array}$ & $\begin{array}{c}\text { Institución } \\
\text { marco }\end{array}$ & Función o cargo \\
\hline E01 & $\mathrm{CICE}^{*}$ & $\begin{array}{l}\text { Unicen (rectorado) } \\
\text { Pública }\end{array}$ & Colaborador externo \\
\hline $\mathrm{E} 02$ & Club de Teatro & Privada & Titular de la institución \\
\hline E03 & $\mathrm{ISFD}^{* *} 10$ & Pública & $\begin{array}{l}\text { Docente de la Tecnicatura en } \\
\text { Gestión Cultural }\end{array}$ \\
\hline E04 & ISFD 10 & Pública & Director \\
\hline E05 & Multimedios El Eco & Privada & $\begin{array}{l}\text { Periodista y responsable del Área } \\
\text { de Cultura y Sociales }\end{array}$ \\
\hline E06 & Subsecretaría de Cultura y Educación & Municipalidad de Tandil & Subsecretaria \\
\hline E07 & Teatro La Fábrica & $\begin{array}{l}\text { Unicen, Facultad de Arte } \\
\text { Pública }\end{array}$ & $\begin{array}{l}\text { Presidente de la Asociación Amigos } \\
\text { del Teatro La Fábrica (2013) }\end{array}$ \\
\hline E08 & CICE & $\begin{array}{l}\text { Unicen } \\
\text { (rectorado) } \\
\text { Pública }\end{array}$ & Coordinador \\
\hline E09 & Club de Teatro & Privada & Titular de la institución \\
\hline E10 & PAT ${ }^{*+*}$, nodo Buenos Aires & $\begin{array}{l}\text { Unicen } \\
\text { (rectorado) } \\
\text { Pública }\end{array}$ & Asistente de gestión en la provincia \\
\hline Ell & Secretaría de Extensión & $\begin{array}{l}\text { Unicen } \\
\text { (rectorado) } \\
\text { Pública }\end{array}$ & Coordinadora de Cultura y Arte \\
\hline
\end{tabular}




\begin{tabular}{|c|c|c|c|}
\hline $\begin{array}{l}\text { Código de } \\
\text { entrevistado }\end{array}$ & $\begin{array}{c}\text { Institución a la que } \\
\text { pertenece }\end{array}$ & $\begin{array}{c}\text { Institución } \\
\text { marco }\end{array}$ & Función o cargo \\
\hline $\mathrm{El} 2$ & PAT, nodo Buenos Aires & $\begin{array}{l}\text { Unicen } \\
\text { (rectorado) } \\
\text { Pública }\end{array}$ & Coordinadora \\
\hline $\mathrm{E} 13$ & Facultad de Arte & $\begin{array}{l}\text { Unicen } \\
\text { Pública }\end{array}$ & Decano \\
\hline \multicolumn{4}{|c|}{ * Centro de Innovación y Creación de Empresas. } \\
\hline \multicolumn{4}{|c|}{${ }^{* *}$ Institutos Superiores de Formación Docente. } \\
\hline *** Polo Audiovisual Tec & gico. & & \\
\hline
\end{tabular}

\section{Descripción de las redes en análisis}

A continuación se expondrán los resultados de la investigación a través de la metodología de redes. La red resulta de la combinación de todas las menciones de los individuos de la ola cero de información o de colaboración para llevar adelante una alianza. En el análisis de los indicadores, se debe tener presente que este estudio abarca la ola cero y uno de las mencionadas redes.

La red resultante se compone de 147 individuos, 13 pertenecen a la ola cero, y los 134 restantes a la ola uno. Entre ellos sostienen 206 lazos, por lo que se puede calcular una densidad de $1 \%$ (baja conectividad) dentro de ella.

En términos prácticos, y sin dejar de considerar que este trabajo recibe la información de la ola cero y uno, resulta una red de baja densidad donde se espera encontrar la presencia de agujeros estructurales, lo que se puede considerar una de las fuentes de capital social. En las figuras 2 y 3 se puede observar la ausencia de vínculos directos entre zonas densas de la red (como las relaciones que nacen de E10 y de E06).

La subred está compuesta por las menciones realizadas por E10 (asistente de gestión del PAT), las cuales corresponden principalmente a personas del ámbito de las producciones audiovisuales, ya sean integrantes del Programa Nacional Polos, del equipo de trabajo de la Productora de Contenidos de la Unicen, o realizadores audiovisuales independientes de Tandil y de otras localidades de la provincia. Esta subred está "intermediada" por E01 (colaborador del CICE) y n101 (integrante de la Productora de Contenidos de la Unicen), con la subred de E03 (docente de la Carrera 
de Gestión Cultural del ISFD 10), compuesta por personas relacionadas con el ámbito de la gestión cultural y el teatro independiente de la ciudad.

Este ejemplo sirve para ilustrar que dos grupos de individuos de dos sectores distintos se vinculan indirectamente $y$, a su vez, permiten la circulación de conocimiento y recursos novedosos y no redundantes.

En la figura 2, se muestra la red conjunta, compuesta por los vínculos de información y las alianzas, llevados adelante por parte del sector. Los únicos lazos que se diferencian de los demás (con grosores diferentes) son los que unen a los entrevistados con las personas con quienes dicen compartir tanto información como colaboración (por ejempplo E12 con E10 mantiene ambas relaciones -línea gruesa- y con E1 una sola de las relaciones -línea delgada-).

Analizando la centralidad de grado dentro de la misma red, E13 (decano de la Universidad de Arte) es el actor más mencionado dentro de la red (29 menciones) por los considerados individuos clave. E05 (periodista responsable del Área de Cultura y Sociales del Multimedios El Eco) es mencionado por 26 individuos y E10 (asistente en la provincia del PAT del nodo Buenos Aires) por 24. Se puede decir que estos tres actores son los de mayor centralidad dentro de esta red.

Por su parte, de los actores resultantes que conforman la ola uno, el actor n039 es quien recibe mayor grado de centralidad, con cinco menciones. El actor de la ola cero que menor número de menciones recibe es E06 (subsecretaria de Cultura y Educación de la Municipalidad de Tandil), quien recibe solo cinco menciones. Este valor, comparativamente, resulta bajo por formar parte de los actores de la ola cero y a priori considerarse un actor institucional clave. 


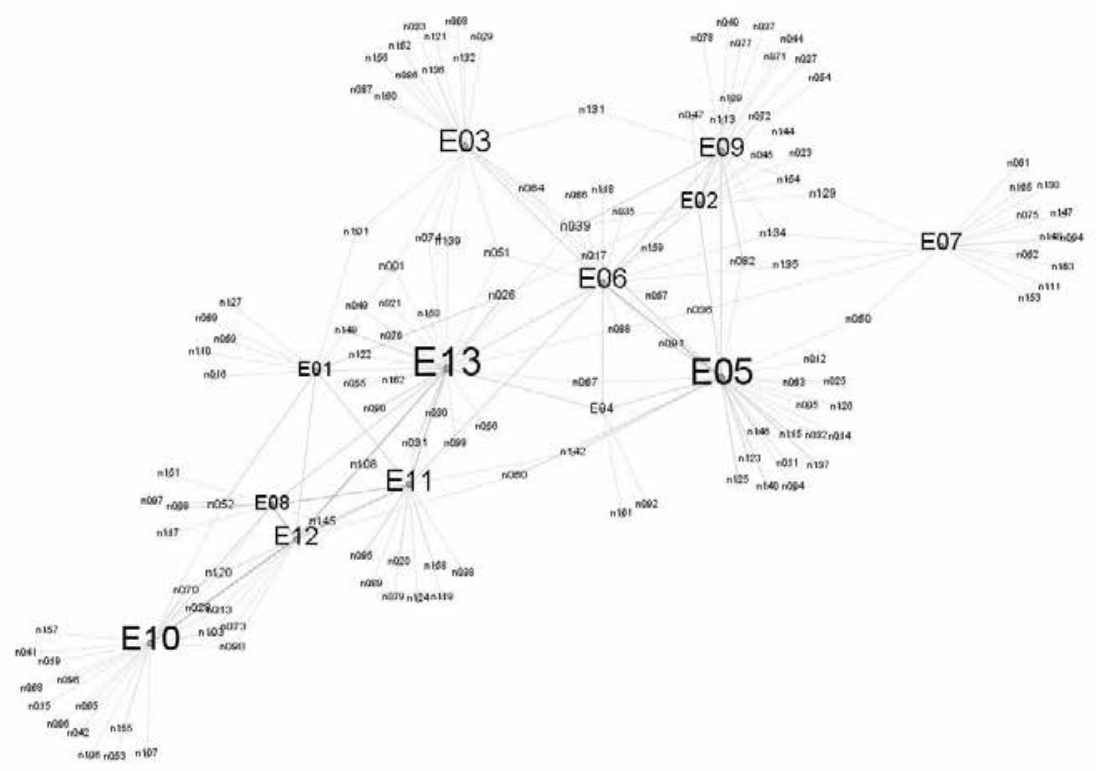

Figura 2. Red de información y alianzas. Centralidad de grado.

En la Figura 3 se analiza el grado de centralidad por intermediación, donde se calcula la participación de los nodos en caminos existentes dentro de la red. El actor E11 (coordinadora de Cultura y Arte de la Unicen) es el individuo que más actores conecta (interviene en 427 caminos dentro de la red), luego le sigue E06 (subsecretaria de Cultura y Educación de la Municipalidad de Tandil), quien participa de 383, y en tercer lugar E13 (decano de la Universidad de Arte) con 270 intervenciones.

Es importante identificar que los actores E01, E04 y E07, si bien forman parte de la ola cero, y fueron considerados a priori como actores clave dentro de la red, a partir del estudio realizado se puede afirmar que estos no alcanzan a conectar ningún par de actores dentro de la red de información y alianza. 


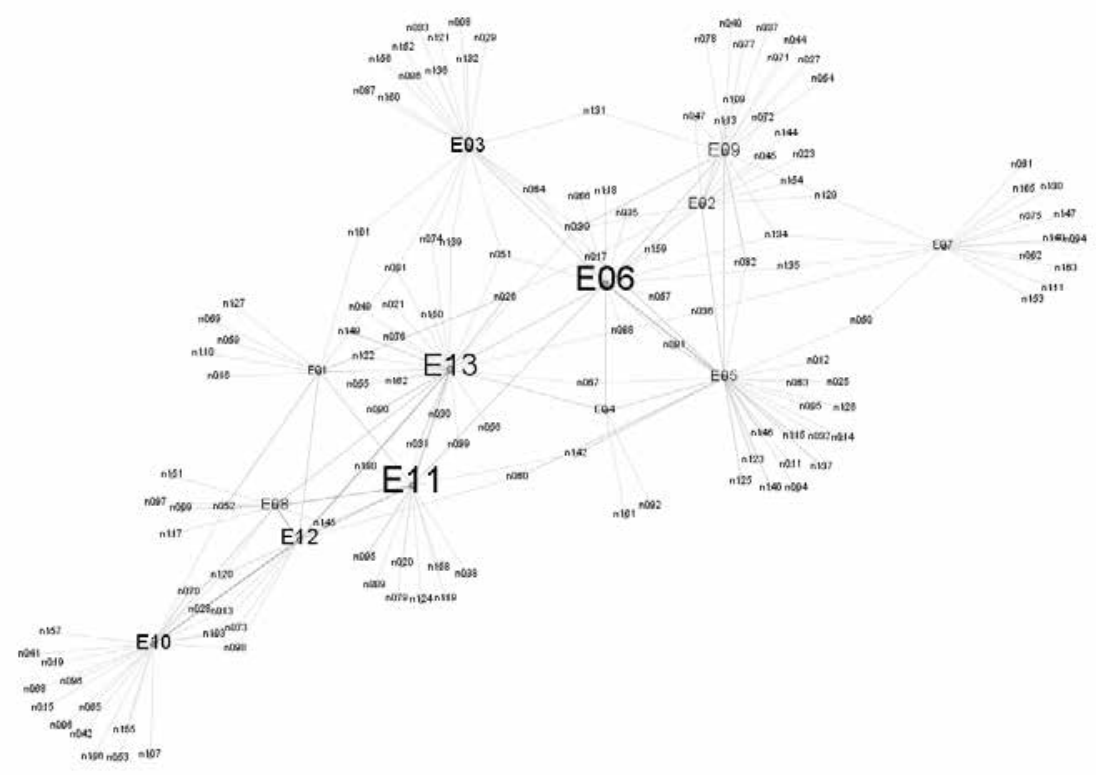

Figura 3. Redes de información y alianzas. Centralidad de intermediación.

\section{Barreras e impulsores de la innovación en el Polo de Industria Cultural y Artes Creativas}

Para identificar actores clave en el condiciona el nacimiento y desarromomento de impulsar iniciativas y lo de las dos carreras que se dictan generar vínculos para procesos de (Teatro y Realización Audiovisual). innovación sostenidos, en las entrevistas en profundidad se hizo énfasis en cuestiones referidas a los papeles de las instituciones a las que pertenece cada entrevistado, además de las vinculaciones con otras organizaciones y maneras de impulsar el desarrollo de la innovación en el clúster en estudio.

Para E13, decano de la Facultad de Arte, la historia de la Facultad

De las hipótesis citadas en el marco teórico (Lazzaretti et al., 2009), la formación de clústeres en las industrias creativas en el caso del Polo de Industria Cultural y Artes Creativas se puede vincular con la presencia de herencia histórica y cultural.

El decano de la citada facultad destaca tres necesidades importantes para el desarrollo del sector: la generación de emprendedores, el 
desarrollo de la demanda y la creación de mayores espacios de diálogo para fomentar la articulación con el municipio. Estos pueden entenderse como puntos de palanca para la generación de innovación.

También resalta factores contextuales positivos para el desarrollo tecnológico de las artes audiovisuales, como los bajos costos y la globalización. En cuanto al teatro, identifica un cambio en la dinámica de la actividad local, pasando de ser dominado por "grupos estables" a "espectáculos concertados", en los cuales en cada obra se articulan las personas a trabajar en ella, con la presencia de actores y directores referentes.

E12, coordinadora del nodo Buenos Aires del Programa Polos, acentúa la necesidad de generar una noción de industria-mercado. Destaca la relación de la productora con la universidad, y la importancia para el Programa Polos de la organización, gestión y sobre todo de vínculos de confianza. La confianza es clave para el desarrollo de capital social y favorece la sostenibilidad de las relaciones.

La representante del municipio (E10) subraya dos iniciativas de colaboración llevadas a cabo por este, además de un acuerdo de trabajo con el Club de Teatro. Identifica la función propia del municipio (promoción de los valores y desarrollo de la cultura), y la complementariedad con los papeles de formación que cumple la universidad. Destaca también la falta de vinculación con el Programa Polos.

Por su parte, E02 y E09 (dueñas del Club de Teatro - el emprendimiento privado más exitoso de la ciudad-) señalan el apoyo del municipio para el desarrollo de su empresa.

E01 y E08 (integrantes del CICE) destacan la incubadora que se creó entre su institución y la Facultad de Arte para apoyar y generar proyectos artísticos y culturales, llamada SustentArte. También identifican la necesidad de estar al tanto de avances tecnológicos en las artes y la importancia de la generación de clústeres para el desarrollo de nuevas empresas y emprendimientos. Se requiere mayor apertura en el ámbito del Polo de Televisión Digital e interacción con otros actores institucionales extrazona.

Para E11 (del equipo de la Secretaría de Extensión de la Unicen), el trabajo artístico es cooperativo, se articulan individuos o pequeños productores. Señala el papel del Programa Polos en la elección y el acompañamiento de proyectos. Identifica la importancia de "pertenecer" y "participar" y el 
armado de las redes para viabilizar los proyectos. Destaca la iniciativa del Festival de Cine como un evento importante en el sector. También identifica el papel de la incubadora SustentArte en el desarrollo del emprendimiento de los actores y directores del sector audiovisual, a la vez que señala algunos referentes del sector.

\section{Conclusiones y discusión}

A partir de entender que el estudio de la innovación puede realizarse desde dos instancias (Borello y González, 2013), el conjunto productivo o fragmentos de este conjunto, y en las empresas individuales, este trabajo se focalizó en la primera de estas dos instancias mencionadas.

La construcción de un mapa de relaciones de los actores clave del Polo de Industria Cultural y Artes Creativas se justifica a partir de destacar la importancia de los clústeres regionales para impactar positivamente respecto de ideas innovadoras, de vinculación con centros especializados de investigación y desarrollo, además de considerar que la interacción social facilita el desarrollo de vínculos estrechos o capital social (Nahapiet y Ghoshal, 1998).

Esta perspectiva de la innovación guarda relación con la llamada innovación abierta (Chesbrough y Garman, 2009) e implica considerar en el análisis tanto el flujo hacia las organizaciones de contribuciones externas (outside-in open innovation), como el proceso de invertir en proyectos generadores de innovación fuera de la organización (insideout open innovation) que promueve ecosistemas innovadores.

En el momento de presentar los aspectos conclusivos de este trabajo, resulta necesario reiterar que incluye las olas cero y uno de una investigación más amplia.

A este punto, resultó posible construir el mapa de relaciones de los actores identificados como claves del Polo de Industria Cultural y Artes Creativas se plantean conclusiones preliminares que contribuyen a la detección de las capacidades existentes para la generación de innovación sostenida en las organizaciones del sector.

$\mathrm{Al}$ tomar como punto de partida a representantes y responsables de instituciones de Tandil, la red resultante denota las vinculaciones existentes entre ellas. Sin olvidar que, si bien se tuvieron en cuenta ocho instituciones consideradas relevantes dentro del sector, ya sea como potenciales articuladores entre emprendedores 
o organizaciones públicas o privadas, están representadas por individuos considerados clave para la generación de cada uno de los lazos mencionados.

En el estudio de los patrones de enlaces entre sus miembros, se realizó una evaluación básica de la estructura de la red social del Polo de Industria Cultural y Artes Creativas, mediante el análisis de tamaño y la densidad de esta red y la centralidad de los actores. Entendiendo que la importancia que un actor tiene en una red puede deberse a su capacidad de controlar la comunicación entre las diversas organizaciones, se estudió la centralidad por intermediación.

Para el diagnóstico de la capacidad de la red para generar innovación sostenida en la dimensión relacional, se hizo hincapié en los vínculos de información existentes en la red y en los relacionados con el potencial asociativo (alianzas para llevar adelante un emprendimiento cultural), a lo que se sumó el análisis de las percepciones manifestadas por los actores identificados como claves en el presente estudio, a partir de los resultados obtenidos en los indicadores de las mencionadas redes.

Del cálculo de la densidad se obtuvo como resultado una baja conectividad dentro de ella; este indicador deberá ser recalculado cuando se completen las olas subsiguientes del presente estudio.

El análisis de centralidad de grado dentro de la red que incluye las relaciones de información y alianzas permite identificar los actores con mayores menciones y analizar su papel institucional.

Se pudo identificar que los actores más relevantes corresponden, en primer término, a un actor institucional que a priori puede considerarse clave: el decano de la Facultad de Arte, y por otra parte, en segundo y tercer lugar a la periodista responsable del Área de Cultura y Sociales del Multimedios El Eco y a la asistente en la provincia del PAT. En estos dos últimos casos, los papeles, aunque en primera instancia no resultan institucionalmente relevantes, sí surgen como actores que pueden ser articuladores importantes del sector en la ciudad.

Se pudo identificar el actor n039, quien recibe mayor grado de centralidad que otros considerados nodales a priori y que formaron parte de la ola cero.

En cuanto al análisis de centralidad por intermediación, surge que el actor E11 (coordinadora de Cultura 
y Arte de la Secretaría de Extensión de la Unicen) es el individuo con más participación en los caminos de la red (427), luego le sigue E06 (subsecretaria de Cultura y Educación de la Municipalidad de Tandil) con 383 caminos, y en tercer lugar E13 (decano de la Universidad de Arte) con 270.

Es de destacar que la subsecretaria de Cultura y Educación de la Municipalidad de Tandil obtiene solo cinco menciones y ocupa el último lugar de los actores de la ola cero respecto de centralidad de grado, aunque el segundo lugar en cuanto al indicador de centralidad por intermediación. Este actor resulta articulador respecto de intermediación, información por tener en cuenta si se quieren propiciar iniciativas que fomenten las alianzas para generar innovación.

Por otra parte, se pudieron identificar actores considerando a priori el potencial de articulación (E01, E04 y E07), y que a partir del estudio realizado se puede afirmar que no llevan adelante ese papel.

Se destacó la importancia de los lazos débiles en los proceso de difusión (de ideas, información, innovaciones, etc.) y de la importancia de los lazos fuertes en procesos de generación de innovación a partir de lo propuesto por Uzzi y Dunlap (2005).
Se pudo graficar (figuras 2 y 3 ) los lazos más fuertes con líneas de mayor grosor representando los que unen a los entrevistados con las personas con quienes dicen compartir tanto información como tener o estar dispuestos a desarrollar relaciones de colaboración para la generación de alianzas.

Se pudo identificar la presencia de agujeros estructurales, lo que se puede considerar una de las fuentes de capital social. En las figuras 2 y 3 se puede observar la ausencia de vínculos directos entre zonas densas de la red (como las relaciones que nacen de E10 y de E06).

Este ejemplo resultó útil para ilustrar que dos grupos de individuos de dos sectores distintos se vinculan indirectamente $y$, a su vez, permiten la circulación de conocimiento y de recursos novedosos y no redundantes, aunque también permite señalar la ausencia de trabajo conjunto y proyectos de cooperación entre los dos sectores (el Programa Polos y la Municipalidad de Tandil).

A partir del análisis realizado, se puede identificar la necesidad de generar lazos transversales y alianzas institucionales, más allá de los personales, que permitan dejar de lado cuestiones políticas, sociales y 
económicas, para el crecimiento y desarrollo del sector y sus actividades.

Todo lo dicho se resume en la existencia de capacidades para la generación y difusión de la innovación, lo cual, de alguna manera, se relaciona con una etapa avanzada dentro del desarrollo embrionario del clúster (sin considerar el financiamiento externo). Avanzar hacia una etapa de crecimiento implicaría, desde el trabajo de la red social y la caracterización de Rosenfeld (2002), buscar los mecanismos para detectar y potenciar a las personas que cumplen papeles de intermediación, así como para ampliar el alcance de la red de manera que incorpore financiamiento externo al polo y atraiga nuevos jugadores.

Por último, es importante considerar este trabajo de investigación como el primer acercamiento al mapeo de organizaciones del sector cultural y arte creativo de Tandil. Los resultados del posterior estudio referido potenciarán las posibilidades de concluir acerca de las consideraciones preliminares vertidas en el presente trabajo.

\section{Referencias bibliográficas}

Altenburg, T. y Meyer-Stamer, J. (1999). How to promote clusters: policy experiences from Latin America.
World Development, 27(9), 16931713.

Bakhshi, H. y Throsby, D. (2009, diciembre). Innovation in arts and cultural organisations. Hamburgo: NESTA.

Borello, J. A. y González, L. (2013). Industrias culturales, innovación y formas de organización en un país semiindustrializado: el caso de la producción audiovisual en la Argentina. En Conferência Internacional LALICS 2013 "Sistemas Nacionais de Inovação e Políticas de CTI para um Desenvolvimento Inclusivo e Sustentável". Río de Janeiro: RedeSist e Instituto de Economia-UFRJ.

Borgatti, S. P. y Halgin, D. S. (2011). On network theory. Organization Science, 22(5), 1168-1181.

Brandes, U. (2001). A faster algorithm for betweenness centrality. Journal of Mathematical Sociology, 25(2), 163 177.

Camio, M. I.; Bricker, A. y Rébori, A. (2012). Desarrollo de capacidades innovativas. La cultura y su relación con el modelo de negocios. Estudio de casos en empresas de software. En 
XVII Reunión Anual de la Red Pymes Mercosur. Río de Janeiro: Mercosur.

Camio, M. I., Rébori, A. y Romero, M. del C. (2009). Cultura y modalidades de gestión: su relación con la innovación. Avances en el desarrollo de marco teórico y la relación entre las variables de análisis. En XIV Reunión Anual de la Red Pymes Mercosur. Santa Fe, Argentina: Mercosur.

Chesbrough, H. W. y Garman, A. R. (2009). How open innovation can help you cope in lean times. Harvard Business Review, 87(12), 68-76.

Corral, M. del (2005). Hacia nuevas políticas de desarrollo de las industrias culturales. Boletín GC: Gestión Cultural, 13. Recuperado de http:// www.gestioncultural.org/boletin/pdf/ bgc13-MCorral.pdf

Cooke P. y Lazzeretti, L. (2008). Creative cities, cultural clusters and local economic development. Edward Elgar, Cheltenham.

Díez, J. I. (2008, junio). Organizaciones, redes, innovación y competitividad territorial: análisis del caso Bahía Blanca. Redes: Revista hispana para el análisis de redes sociales, 14(3).
Recuperado de http://revista-redes. rediris.es/html-vol14/vol14_3.htm

Florida, R. (2002). The rise of the creative class: and how it's transforming work, leisure, community and everyday life. Nueva York: Basic Books.

Gaete Fiscella, J. M. y Vásquez, J. I. (2008). Conocimiento y estructura en la investigación académica: una aproximación desde el análisis de redes sociales. Redes: Revista hispana para el análisis de redes sociales, 14(5). Recuperado de http://revista-redes. rediris.es/html-vol14/vol14_5.htm

García Domínguez, B. (2010). Tesina: clústeres de industrias creativas en España. Barcelona: Universidad Autónoma de Barcelona.

García Macías, A. (2002). Redes sociales y clústeres empresariales. Redes: Revista hispana para el análisis de redes sociales, 1(6), 1-20.

Getino, O. (2004, febrero). La cultura como capital. En Asamblea Internacional de Economistas. La Habana: ANEC. Recuperado de http://octaviogetinocine.blogspot.com.ar/2010/06/ la-cultura-como-capital.html 
Gómez Minujín, G. (2005). Competitividad y complejos productivos: teoría y lecciones de política. Serie Estudios y Perspectivas, 27. Buenos Aires: Cepal.

Granovetter, M. S. (1973). The strengh of weak ties. American Journal of Sociology, 78(6), 1360-1380.

Hanneman, R. A. (2001). Introduction to social network methods. Riverside, CA: University of California.

Helfat, E. et al. (2007). Dynamic capabilities: understanding strategic change in organizations. Malden, MA: Blackwell Publishing.

Kind, S. y Meier zu Köcker, G. (2013). Developing successful creative \& cultural clusters. Measuring their outcomes and impacts with new framework tools. IIT Publication.

Koepp, R. (2002). Clusters of creativity: enduring lessons on innovation and entrepreneurship from Silicon Valley and Europe's Silicon Fen. Chichester, UK: John Wiley \& Sons.

Lazzeretti, L.; Boix, R. y Capone, F. (2009). Why do creative industries cluster? An analysis of the determinants of clustering of creative industries. Iermb Working Paper in Economics, 9(2), 1-30.

Leeuwis, C. y Aarts, N. (2011). Rethinking communication in innovation processes: creating space for change in complex systems. The Journal of Agricultural Education and Extension, 17(1), 1-13.

Lozares Colina, C. (1996). La teoría de redes sociales. Papers: Revista de Sociología, 48, 103-126. Recuperado de http://ddd.uab.cat/pub/papers/0 2102862n48/02102862n48p103.pdf

Lundvall, B. y Maskell, P. (2000). Nation states and economic development: from national systems of production to national systems of knowledge creation and learning. En G. Clark, J. Nahapiet y M. Gertler (eds.), The Oxford handbook of economic geography (pp. 353-372). Oxford: Oxford University Press.

Mayoral, L. et al. (2012, julio-diciembre). Red de mentoría emprendedora en el sector de las tecnologías de la información y la comunicación: la diversidad de los roles sociales y la fuerza de los lazos débiles como soportes de la innovación tecnológica en 
Tandil, Argentina. Revista Cuadernos de Administración, 25(45), 137-162.

Miles, I. y Green, L. (2008). Hidden innovation in the creative industries. Hamburg: NESTA.

Nahapiet, J. y Ghoshal, S. (1998). Social capital, intellectual capital and the organizational advantage. The Academy of Management Review, 23(2), 242-266.

Porter, M. E. (1998, noviembrediciembre). Clusters and the new economics of competition. Harvard Business Review, 75-90.

Power, D. (2011). Priority sector report : creative and cultural industries. Europa Innnova, 16.

Rosenfeld, S. (2002). Creating smart systems: a guide to clusters strategies in less favored regions. En Informe para la Unión Europea-Regional Innovation Strategies, 6. Carboro, North Carolina: Regional Technology Strategies.

Saxenian, A. (1994). Regional advantage: culture and competition in Silicon Valley and Route 128. Cambridge, MA: Harvard University Press.
Scott, A. J. 2005. On Hollywood: The place, the industry. Princeton: Princeton University Press.

Stoneman, P. (2010). Soft innovation. Economics, product aesthetics, and the creative industries. Nueva York: Oxford University Press Inc.

Teece, D. (2007). Explicating dynamic capabilities: the nature and microfoundations of (sustainable) enterprise performance. Strategic Management Journal, 28(13), 1319-1350.

Unctad (2008). Creative Economy Report. The Challenge of Assessing the Creative Economy: Towards Informed Policy-making. Nueva York: Naciones Unidas.

Uzzi, B. (1997). Social structure and competition in interfirm networks: the paradox of embeddedness. Administrative Science Quarterly, 42(1), 35-67.

Uzzi, B. y Dunlap, S. (2005). How to build your network. Harvard Business Review, 1-9.

Velázquez Álvarez, O. A. y Aguilar Gallegos, N. (2005). Manual introductorio al análisis de redes sociales: 
medidas de centralidad. México: Universidad Autónoma del Estado de México y Universidad Autónoma de Chapingo.

Verd Pericás, J. M. y Martí Olivé, J. (1999). Muestreo y recogida de datos en el análisis de redes sociales. Questiió: Quaderns d'Estadística, Sistemes, Informatica i Investigació Operativa, 23(3), 507-524.

Wasserman, S. y Faust, K. (1994). Social network analysis: methods and applications. Cambridge: Cambridge University Press.

Ying, T. (2010). Social networks in the tourism industry: an investigation of Charleston. South Carolina, SCA: Clemson University.

Yoguel, G. y Boscherini, F. (1996). La capacidad innovativa y el fortalecimiento de la competitividad de las firmas: el caso de las pymes exportadoras argentinas. Documento de Trabajo, 71, Cepal. 